\title{
Meta
}

Journal des traducteurs

Translators' Journal

\section{LÉPINETTE, B. y A. MELERo (eds.) (2003) : Historia de la traducción, Valencia, Quaderns de Filología, Estudis Lingüístics VIII, Universitat de València, 311 p.}

\section{Georges L. Bastin}

Volume 50, numéro 3, août 2005

Le prisme de l'histoire

The History Lens

URI : https://id.erudit.org/iderudit/011614ar

DOI : https://doi.org/10.7202/011614ar

Aller au sommaire du numéro

Éditeur(s)

Les Presses de l'Université de Montréal

ISSN

0026-0452 (imprimé)

1492-1421 (numérique)

Découvrir la revue

Citer ce compte rendu

Bastin, G. L. (2005). Compte rendu de [LÉPINETTE, B. y A. MELERo (eds.) (2003) :

Historia de la traducción, Valencia, Quaderns de Filología, Estudis Lingüístics

VIII, Universitat de València, 311 p.] Meta, 50(3), 1051-1053.

https://doi.org/10.7202/011614ar d'utilisation que vous pouvez consulter en ligne. 


\title{
Comptes rendus
}

\author{
Lépinette, B. y A. Melero (eds.) (2003): Historia de la traducción, Valencia, Quaderns \\ de Filología, Estudis Lingüístics VIII, Universitat de València, 311 p.
}

L'Espagne, on l'a appris au cours des dernières années, est sans doute le pays dont la production historiographique en traductologie est la plus abondante au monde. Il convient dès lors de scruter chacune de ces nouvelles publications pour y trouver ce qui la distingue des précédentes. Les études de cas peuvent évidemment être multipliées à satiété et remplir les rayons d'une bibliothèque. Les chercheurs et les amateurs trouvent dans celles-ci le matériau qui permet de dépasser le travail purement descriptif - combien difficile et nécessaire. C'est ainsi que l'on commence à voir de plus en plus de travaux dépassant l'étape purement archéologique pour donner des explications, voire des interprétations, du rôle de la traduction et des traducteurs dans l'histoire.

Brigitte Lépinette, auteure de l'une des très rares études méthodologiques ${ }^{1}$, et Antonio Melero, prix national de traduction en 1997, tous deux professeurs à l'Université de Valencia, offrent dans ce volume une variété d'articles qui reflètent diverses facettes de la recherche historique en traductologie. D'où vraisemblablement la simplicité du titre du recueil: Historia de la traducción, sans plus. Dans les faits, il s'agit de douze articles consacrés à l'analyse de textes traduits en espagnol, durant la période allant de la Renaissance au XIX ${ }^{\mathrm{e}}$ siècle, d'une langue européenne occidentale et, dans un cas, des langues latine, grecque, perse et arabe.

Avant de détailler ces études, il convient d'en souligner le fil conducteur non explicitement avoué: la méthodologie de la recherche en historiographie de la traduction. Les auteurs affirment d'ailleurs dans leur présentation: "L'historiographie traductologique en tant que telle n'a suscité qu'un nombre très réduit de réflexions méthodologiques» et «n'est pas le résultat de l'apparition d'un texte fondateur de la discipline qui aurait situé cette branche de l'histoire dans l'ensemble des sciences connexes » (p. xi, notre traduction). C'est pourquoi Lépinette reprend, dans cette présentation, une partie de sa caractérisation des objets et des méthodes en historiographie traductologique ${ }^{2}$. Si elle reprend sa réflexion c'est pour l'étayer de ses observations ultérieures et surtout pour l'illustrer par des exemples éloquents sur lesquels nous reviendrons. Son but: poser les conditions du travail de recherche en histoire de la traduction. Rappelons ${ }^{3}$ que Lépinette distingue deux modèles de base «soit le modèle sociologique-culturel et le modèle historique-descriptif». Le premier "prend en compte le contexte social et culturel [...] d'un phénomène (en l'occurrence la traduction) au moment de sa production et à celui de sa réception» (p. 101). L'objet de ce premier modèle est le péritexte, à savoir "tous les événements et phénomènes qui accompagnent la production d'un texte ou ensemble de textes traduits et son apparition dans un contexte socio-culturel récepteur qui détermine les caractéristiques de la traduction et permettra d'expliquer son influence» (p. 101). Le second, historique-descriptif, se subdivise en deux sousmodèles, descriptif-comparatif et descriptif-contrastif, chacun avec ses objets et ses techniques d'analyse propres. Le descriptif-comparatif traite les métatextes traductologiques pour analyser les concepts métatraductologiques. Il portera notamment sur l'évolution d'un même concept métatraductologique dans divers textes d'époques différentes ou sur l'ensemble des concepts métatraductologiques d'un même texte. Le descriptif-contrastif «se fonde sur les 
options choisies par les traducteurs d'un texte cible ou sur une série de textes cibles correspondant à un même texte source» (p. 103). "Pour réaliser une telle description, le chercheur adopte une approche globale, textuelle, qui envisage le texte original comme un tout comparé $\mathrm{au}(\mathrm{x})$ texte(s) cible(s) à un niveau macro ou microtraductologique. Il peut aussi suivre une approche sélective, linguistique, qui le fera sélectionner un élément important du texte original pour le contraster dans le(s) texte(s) traduit(s). Voilà qui unit très intimement l'histoire de la traduction à celle de la langue et de la littérature.»

C'est une illustration de ces divers modèles que Lépinette et Melero donnent avec les différentes études présentées. Les deux premiers sont des exemples types de travaux archéologiques. Le premier, «Un quehacer olvidado: los intérpretes-traductores de navíos», de Julio César Santoyo, étudie «les interprètes quasi anonymes de la marine marchande». Après une brève incursion dans le monde des premiers interprètes improvisés de la conquête américaine, Santoyo extrait des archives historiques du Pays Basque des données qui réhabilitent cette catégorie professionnelle d'intermédiaires socioculturels: le profil linguistique et moral de ses membres et leur statut administratif et institutionnel. Le deuxième, de H. J. Niederehe, appartient plutôt à l'archéologie lexicographique à propos de la généalogie d'un Vocabulaire catalan-allemand et allemand-catalan publié en 1502, mais dont le modèle italien-allemand remonte à 1477. C'est aussi l'histoire des premiers dictionnaires de langues «modernes» qui se distinguent des classiques du latin, des dictionnaires dont le public est davantage mercantile et dont l'objet est, outre la traduction, l'enseignement de la langue.

Issus du modèle sociologique-culturel (péritexte), cinq articles. J. Gómez de Entería délimite un «corpus de traducteurs et d'ouvrages traduits en science et en technique» durant l'Illustration espagnole, analyse les domaines de spécialité, les causes et les conséquences de ces versions et leur insertion dans l'Histoire de la science en Espagne. L'essai, intitulé «Notas sobre la traducción científica y técnica en el siglo XVIII », se termine sur la mise en valeur de cette transmission d'informations scientifiques et techniques. L'article de Brigitte Lépinette, «Traduction et histoire», en français, est une réflexion sur les implications sociologiques et culturelles des traductions. Elle procède d'abord à une longue et richissime introduction de nature méthodologique, dans laquelle elle décrit les trois étapes de la recherche historiographique: 1 . «rendre compte du contexte historique socioculturel et intellectuel dans lequel la question de la traduction [...] se pose»; 2. prendre en compte le processus de traduction lui-même; et 3 . prendre en compte le contexte historique socioculturel et intellectuel dans lequel vient s'insérer l'œuvre traduite. Son corpus appartient aux versions et rééditions espagnoles de textes d'histoire français: Bossuet, Rollin, Mabillon, Le Nain de Tillement, Fleury, Voltaire et, en particulier Joinville. Les deux articles suivants sont des travaux de catalogage de traductions, «base obligatoire des travaux [sociologiques-culturels]» (p. xxi). $M^{a}$ de las Nieves Muñiz présente un répertoire en espagnol des traductions littéraires italiennes au XIX ${ }^{\text {e }}$ siècle; A. Sierra Soriano, pour sa part, offre un catalogue de traductions espagnoles d'œuvres françaises dans le domaine mal connu de l'art militaire. Julio Calvo Pérez scrute les traductions de José Antonio Conde (1766-1820) d'œuvres perses et arabes pour mettre en valeur non seulement les options du traducteur, mais surtout les difficultés de transmettre à l'Europe les valeurs de la culture orientale.

Issu du modèle descriptif-comparatif (métatexte), l'article de Francisco Lafarga sur les traductions de José Marchena revisite les essais qui portent sur l'œuvre du célèbre traducteur, mais se centre sur la pensée traductologique de celui-ci. Les concepts métatraductologiques examinés sont ici en étroite association avec les concepts métalittéraires du XIX ${ }^{\mathrm{e}}$ siècle.

Issus du modèle descriptif-contrastif (binôme textes source et cible), quatre derniers travaux. Cesáreo Calvo Rigual contraste la traduction du Galateo de Giovanni della Casa (1585) par Domingo Becerra avec l'original pour relever les interventions du traducteur, omissions et ajouts, qui visent à naturaliser sa version. Calvo met aussi le doigt sur nombre d'erreurs commises par le traducteur. Ensuite, les linguistes Flor Bango et $\mathrm{M}^{\mathrm{a}}$ Luisa Donaire 
se penchent, toujours selon une approche contrastive et selon la théorie de l'argumentation de Anscombre et Ducrot, sur les concessives en français et en espagnol à partir d'un corpus constitué de Madame Bovary et de La Regenta, et de leurs traductions. Julia Pinilla, de son côté, étudie, dans un texte rédigé en français, la traduction des termes techniques au $\mathrm{XVIII}^{\mathrm{e}}$ siècle et en particulier les couples synonymiques. Finalement, E. Llácer Lorca et N. Estévez se tournent vers l'analyse de la fonction rythmique dans la traduction des Tales d'Edgar Allan Poe par Carlos Olivera (1884) pour y déceler des «zones d'inéquivalences phonétiques et syntaxiques».

Cet ouvrage, au-delà de la richesse et de l'abondance des données archéologiques historiographiques dans le contexte européen du $\mathrm{XvI}^{\mathrm{e}}$ au $\mathrm{XIX}^{\mathrm{e}}$ siècle, constitue un essai unique sur la méthodologie de l'historiographie traductologique. Il mériterait d'être traduit pour le plus grand bénéfice des historiographes traductologiques.

Georges L. Bastin

Université de Montréal, Montréal, Canada

\section{NOTES}

1. Lépinette, Brigitte (1997): La historia de la traducción. Metodología. Apuntes bibliográficos. Lynx, Vol. 14.

2. Op. cit.

3. Voir notre recension de López Alcalá, Samuel. La historia, la traducción y el control del pasado dans META 47-1, p. 132-134.

Lafarga, F. y L. Pegenaute (eds.) (2004): Historia de la traducción en España, Salamanca, Editorial Ambos Mundos, 872 p.

S'il existait encore le moindre doute quant au pays le plus prolifique en matière de publications en histoire de la traduction, il est désormais dissipé: c'est l'Espagne. L'ouvrage de Lafarga et Pegenaute en est un témoin éloquent: avec ses 872 pages, il vient couronner une production que l'on peut estimer, sans crainte d'exagération, à plusieurs milliers de pages sur le même sujet: l'histoire de la traduction en Espagne.

La tâche de rendre compte d'un tel «monument » apparaît dès lors comme une mission impossible. Pour un instant, nous avons pensé écrire cette recension de la manière dont Borges avait entrepris la traduction de l'Ulysse de Joyce, c'est-à-dire en ne le lisant pas et en n'en traduisant que les deux dernières pages! Soyons sérieux, mais pourquoi ne pas commencer ce compte rendu par la dernière page:

\section{Achevé d'imprimer sur les presses de l'imprimerie \\ CALATRAVA, dans la très culte cité de \\ Salamanca, le 30 septembre 2004, fête de SAINT JÉRÔME, docteur de l'Église, patron et protecteur des traducteurs}

Voilà qui «traduit» une planification éditoriale implacable ou, qui sait, un pieux mensonge. Quoi qu'il en soit, ce détail accompagne harmonieusement la couverture du livre qui arbore le relief de saint Jérôme dans la chapelle de l'Université de Salamanca.

Les directeurs du volume sont reconnus chez eux, ils n'en sont pas moins connus à l'étranger. Francisco Lafarga et Luis Pegenaute, professeur de philologie française à 